EPJ Web of Conferences 31, 00027 (2012)

DOI: $10.1051 /$ epjconf/20123100027

(C) Owned by the authors, published by EDP Sciences - SIF, 2012

\title{
Boltzmann-Langevin one-body dynamics for fermionic systems
}

\author{
P. NAPOlitani ${ }^{1}$ and M. COlonna ${ }^{2}$ \\ ${ }^{1}$ IPN, CNRS/IN2P3, Université Paris-Sud 11, 91406 Orsay cedex, France \\ 2 INFN-LNS, Laboratori Nazionali del Sud, 95123 Catania, Italy
}

\begin{abstract}
A full implementation of the Boltzmann-Langevin equation for fermionic systems is introduced in a transport model for dissipative collisions among heavy nuclei. Fluctuations are injected in phase space and not, like in more conventional approaches, as a projection on suitable subspaces. The advantage of this model is to be specifically adapted to describe processes characterised by instabilities, like the formation of fragments from a hot nuclear system, and by dissipation, like the transparency in nucleus-nucleus collisions.
\end{abstract}

\section{Fluctuations and bifurcations}

Dissipative nucleus-nucleus collisions are a unique probe for the in-medium nuclear interaction at densities away from saturation and at high nucleon momenta. In weakly-excited systems, Pauli-blocking factors of final orbitals largely suppress two-body direct interactions. In a semiclassical framework, the dynamics of such situation can be appropriately described within a Vlasov formalism, where the temporal evolution of the one-body distribution function $f(\vec{r}, \vec{p}, t)$ (function of time $t$, space coordinates $\vec{r}$ and momentum $\vec{p}$ ) in the self-consistent one body field is governed by the effective Hamiltonian $H[f]$. However, for more violent collisions, direct two-body interactions become significant and require to be treated by an additional Boltzmann collision integral $I[f]$. In the Uehling-Uhlenbeck form, this latter is introduced as a continuous-source term $\bar{I}[f]$. Within standard forms of BUU/VUU/BNV formalisms, the additional use of an ensemble-averaged mean field makes the permits unrestricted use, distribution, and reproduction in any medium, provided the original work is properly cited. 
transport model particularly suited for one-body observables (for example the collective flow, or proton spectra), but suppresses bifurcations.

Bifurcations are however crucial in processes characterised by volume instabilities, where fluctuations are the seeds of the formation of several intermediate-mass fragments. A possible approach to this scenario is the employment of a two-body Hamiltonian, as in Molecular Dynamics [1]. As an extension of the one-body dynamics, another approach is the introduction of one more term $\delta I[f]$ which produces Langevin fluctuations. The resulting temporal evolution of the one-body distribution function in a BoltzmannLangevin approach is given by

$$
\partial_{t} f=\{H[f], f\}+\bar{I}[f]+\delta I[f] .
$$

This latter is the method we follow.

Many approaches to the Boltzmann-Langevin equation in nuclear transport models are projections on one suitable subspace, like the coordinate space. In this framework, a fluctuating term can be prepared by associating a Brownian force to a stochastic one-body potential [2] or it can correspond to kinetic equilibrium fluctuations of a Fermi gas [3]. The disadvantage of projection methods is that the amplitude of the fluctuation may have to be adjusted and fluctuations may appear from a specific time on (when equilibrium is attained or when entering spinodal conditions), as an amplification of the most unstable mode.

The approach we follow consists in constructing a stochastic collision term which includes Boltzmann-Langevin fluctuations in phase space, supplemented by a strict treatment of Pauli-blocking factors, in the framework of semiclassical test-particle-based transport models.

\section{Collision statistics and fragment formation}

We construct the Bolzmann-Langevin one body (BLOB) model by using the same semiclassical isospin-dependent mean-field employed in the Stochastic Mean Field (SMF) model [2,4]; this is characterised by an incompressibility of $k_{\text {inf }}=200 \mathrm{MeV}$ (soft) and the potential component of the symmetry energy is given either by a linear term as a function of the density (asystiff), or by a quadratic term, with negative curvature (asy-soft).

In SMF, the collision term is introduced in the standard UehlingUhlenbeck form and fluctuations are injected in a reduced subspace, agitating the spatial density profile. On the other hand, in BLOB fluctuations are implemented in full phase-space, replacing the collision integral by 
the Boltzmann-Langevin approach described in the following. Like in the method introduced in ref. [5], the collision term of BLOB involves entire nucleons. The test-particle method is used and a number of $N_{\text {test }}$ test-particles per nucleon is employed. A 'nucleon' is represented by an agglomerate of $N_{\text {test }}$ test-particles of identical isospin which share the same volume in coordinate and in momentum space, as shown in fig. 1.

The number of attempted collisions is weighted on the probability of satisfying the mean-free-path condition for the two agglomerates of test particles in a given interval of time. Such probability is proportional to the mean density in momentum space, the average relative momentum between the two clouds and the nucleon-nucleon cross section. This latter is divided by a factor $N_{\text {test }}$ in order to keep a correspondence with the Uehling-Uhlenbeck collision term. In fig. 2, SMF and BLOB are used to simulate the same system ${ }^{124} \mathrm{Sn}+{ }^{124} \mathrm{Sn}$ at $50 \mathrm{AMeV}$ and $b=0$. We use here a simplified ver-

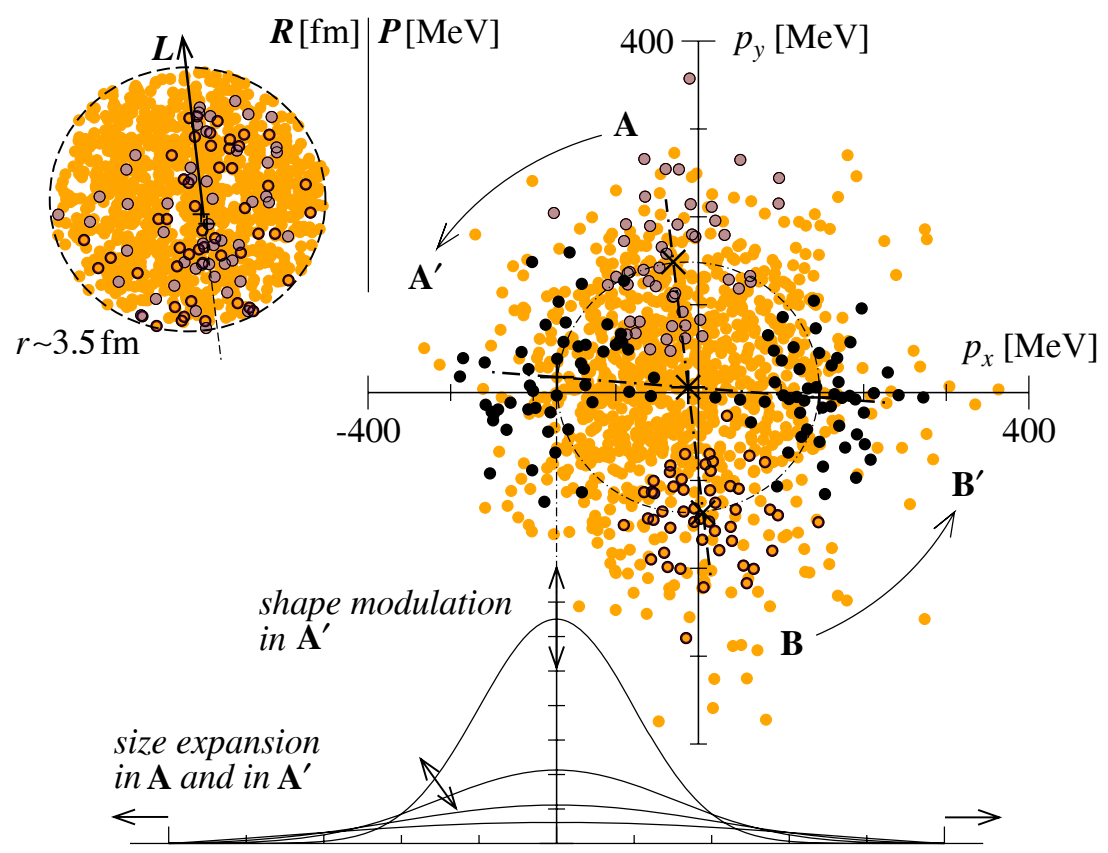

Figure 1: Example of one collision event in BLOB. Two nucleons are represented by two agglomerates of test-particles $A$ and $B$ which share the same volume in coordinate space $\mathcal{R}$. In the corresponding momentum space $\mathcal{P}$ the collision of $A$ and $B$ originates by rotation (determined by the scattering angle) the destination sites $A^{\prime}$ and $B^{\prime}$, where the test-particles are redistributed according to Pauli-blocking and energy constraints. 
sion of the SMF model, where fluctuations in the phase-space mapping are generated by the use of a finite number of test particles, $N_{t e s t}$, in the numerical resolution of Eq. 1. Then, the amplitude of fluctuations injected in the dynamics scales with $1 / N_{\text {test }}$. Several events were simulated so that several trajectories could be drown for the evolution of the number of attempted and effective collisions. Mean trajectory could be deduced as the average of the bundle of all the simulated trajectories, this is shown on the left panel; the right panel shows one single trajectory per type of calculation so as to appreciate the amplitude of the fluctuation. The evolution of the number of collisions as a function of time is a probe of the reaction mechanism. This number increases during the initial stage of the collision, which is a phase of compression. A stage of dilution follows, characterised by a drop of the number of collisions. The trend then reaches a minimum and inverses sign-

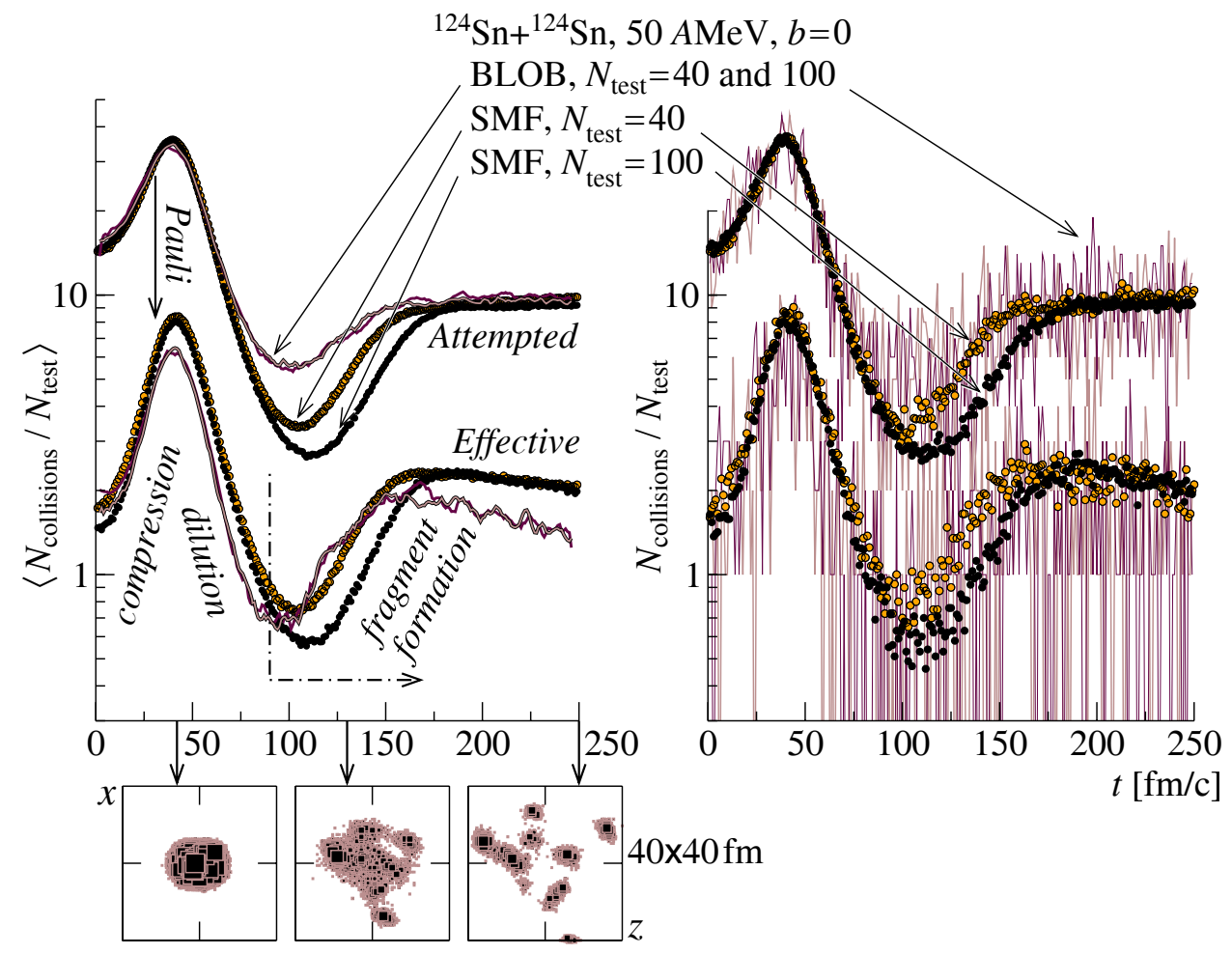

Figure 2: SMF and BLOB compared for the same system ${ }^{124} \mathrm{Sn}+{ }^{124} \mathrm{Sn}$ at $50 \mathrm{AMeV}$ and $b=0$. Left. Mean value of the evolution of the number of collisions extracted from a bundle of trajectories. Right. Amplitude of the fluctuations shown by the evolution of the number of collisions for single events. 
ing the beginning of the process of fragment formation, characterised by an initial increase of the number of collisions and a successive stabilisation and levelling off due to the separation of the fragments. This figure shows that, as expected, the number of attempted collisions per test particle averaged over a statistics of dynamical trajectories should not differ in the present formalism with respect to a conventional BUU approach when the initial rising side of the spectrum is considered. On the other hand, the amplitude of the fluctuation marks the difference between the two models. In correspondence with the phase of dilution (around 80-120 fm/c), fluctuations characterise not only the statistics of collisions but also the kinematics. The simulation was repeated for $N_{t}=40$ and $N_{t}=100$. The incomplete treatment of fluctuations used in SMF results in establishing a dependence of the collision statistics on the numerical parameter $N_{t}$. When $N_{t}$ is reduced in SMF the fragment formation is anticipated, the dynamics becomes more explosive, and the fragment multiplicity increases. All these effects are even further enhanced when moving from SMF with $N_{t}=40$ to BLOB, but in the latter case the dependence on $N_{t}$ disappears, as expected, because in BLOB a fluctuation-source term, corresponding to the Boltzmann-Langevin collision integral, has been implemented.

Like in the approach followed in ref. [6], the effective-collision probability is weighted on the blocking factors of the destination sites, taking into account the full extension of the initial distributions. In BLOB, distributions are adapted to the occupation profile by the use of a numerical optimisation procedure. This latter converges first to the appropriate extension of the scattered regions (by expanding initial and final sites in momentum space) and then converges to the appropriate test-particle final distribution by reorganising the scattered test particles according to packets which can be modulated in shape; the principle of this method is shown in fig. 1. Due to the inclusion of blocking factors in the collision probability, the number of effective collisions drops to smaller values with respect to the number of attempted collisions. The evolution of the effective-collision number is not expected to be identical for SMF and BLOB calculations; the number is reduced with respect to the attempted-collision spectrum, keeping the shape mostly unchanged, but the effective collisions result more penalised by the Pauli blocking treatment in BLOB then in SMF. On the one hand, this difference comes from the fact that the Pauli blocking is applied to the scattering of test-particle couples in SMF, while in BLOB it is applied to extended distributions of test particles. On the other hand, it is important to remark that in BLOB the number of effective collisions is reduced with respect to SMF but in this latter case collisions are more efficient in pro- 
ducing phase-space fluctuations. Moreover, in the BLOB calculation the fluctuation is not gradually amplified (the SMF approach results in amplifying the most unstable mode) but it presents large and correct amplitudes since the initial instants: this favours the early appearing of bifurcations and explains the earlier fragment formation and the larger kinetic energy of the fragments. As a further outcome, a larger variety of exit-channel configurations are expected.

In fig. 3, like in fig. 2, SMF and BLOB are again used to simulate the system ${ }^{124} \mathrm{Sn}+{ }^{124} \mathrm{Sn}$ at $50 \mathrm{AMeV}$ and $b=0$. In full agreement with the analysis of the collision statistics and the properties of fragments, we find larger fragment kinetic energies for the BLOB simulation. The fragment configuration is more isotropic in the BLOB simulation, this could be due to a larger transparency. A similar comparison was done between SMF and
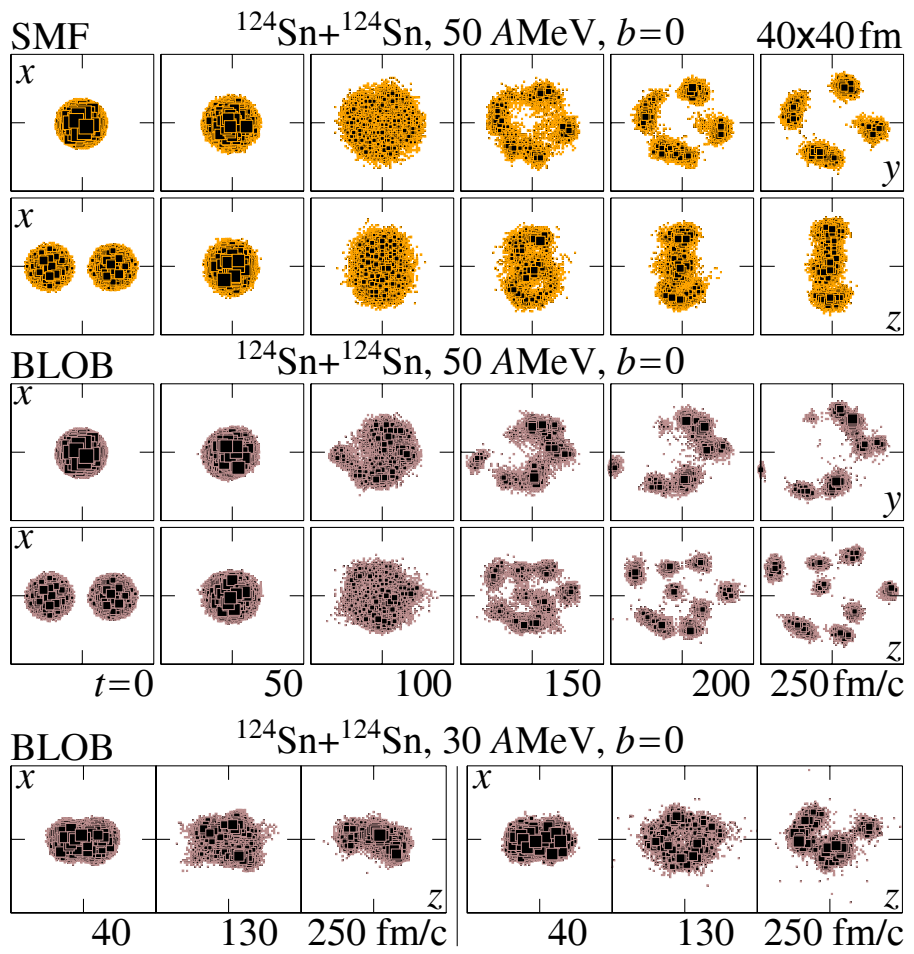

Figure 3: First and second rows: SMF and BLOB compared for the same system ${ }^{124} \mathrm{Sn}+{ }^{124} \mathrm{Sn}$ at $50 \mathrm{AMeV}$ and $b=0 . N_{t}=100$ is used. Third row: Two different exit channels for the same system ${ }^{124} \mathrm{Sn}+{ }^{124} \mathrm{Sn}$ at $30 \mathrm{AMeV}$ and $b=0$, calculated with BLOB. 
AMD in ref. [7].

Another calculation is shown in fig. 3 for the less excited system ${ }^{124} \mathrm{Sn}+{ }^{124} \mathrm{Sn}$ at $30 \mathrm{AMeV}$, close to the threshold between fusion and fragmentation. This analysis has the purpose of studying the effect of bifurcation. In the present example we find that in the SMF calculation one trajectory prevails (leading to fusion), while in the BLOB calculation a more complete phase-space sampling allows to access a variety of exit-channel configurations (from fusion to fragmentation).

\section{Conclusions}

The differences between BLOB and SMF appear in the statistics of collisions as well as in the kinematics.

In this context, the study of a new strategy to solve the BoltzmannLangevin equation is presented. As an extension of the Bauer-Bertsch approach, in the framework of semiclassical test-particle-based transport models, fluctuations of correct amplitude are introduced in phase space through a stochastic collision term. The resulting fluctuations have so large amplitude to induce bifurcations in the dynamical paths of the one-body phase-space density and they correlate over smaller volumes with respect to previous forms of the collision integral. In prospective, this strategy is promising for the improvement of transport models for nuclear collisions and for the implementation of isovector effects in the collision dynamics.

\section{References}

[1] J. Aichelin, Phys. Rep. 202 (1991) 233;

A. Ono, Phys. Rev. C 59 (1999) 854.

[2] A. Guarnera et al. Phys. Lett. B403 (1997) 191.

[3] M. Colonna et al. Nucl. Phys. A642 (1998) 449.

[4] Ph. Chomaz, M. Colonna and J. Randrup, Phys. Rep. 389 (2004) 263.

[5] W. Bauer and G.F. Bertsch, Phys. Rev. Lett. 58 (1987) 863

[6] J. Rizzo, Ph. Chomaz and M. Colonna, Nucl. Phys. A (2008).

[7] J. Rizzo, M. Colonna, A. Ono, Phys. Rev. C 76 (2007) 024611;

M. Colonna, A. Ono, J. Rizzo, Phys. Rev. C 82 (2010) 054613. 